\title{
Notch Signal Regulates Corneal Endothelial-to-Mesenchymal Transition
}

\author{
Cheng $\mathrm{Li}^{* \dagger}{ }^{\dagger \dagger}$ Fei Dong, ${ }^{* \dagger}$ Yanni Jia, ${ }^{* \dagger}$ Huiyi Du, ${ }^{* \dagger}$ Nuo Dong, ${ }^{\ddagger}$ Yajie $\mathrm{Xu},{ }^{* \dagger}$ Shen Wang, ${ }^{* \dagger}$ Huping Wu, ${ }^{\ddagger}$ Zuguo Liu, ${ }^{* \dagger}$ and \\ Wei $\mathrm{Li}^{* \dagger \ddagger}$
}

From the Eye Institute of Xiamen University, * Xiamen; the Fujian Provincial Key Laboratory of Ophthalmology and Visual Science, ${ }^{\dagger}$ Xiamen; and the Xiamen University Affiliated Xiamen Eye Center, ${ }^{\ddagger}$ Xiamen, China

Accepted for publication May 31, 2013.

Address correspondence to Zuguo Liu, M.D., Ph.D., and Wei Li, M.D., M.Sc., Eye Institute of Xiamen University, Medical College of Xiamen University, Xiamen University Affiliated Xiamen Eye Center, Fujian Provincial Key Laboratory of Ophthalmology and Visual Science, South Xiang'an Road, Xiamen, Fujian 361102, China. E-mail: zuguoliu@xmu. edu.cn orwei1018@gmail.com.

\begin{abstract}
Endothelial-to-mesenchymal transition (EnMT) is a cell transformation process involved in both morphogenesis and pathogenesis. EnMT of corneal endothelial cells happens after endothelial injury and during ex vivo culture. Previous studies have shown that the transforming growth factor- $\beta$ signaling pathway is involved in this transition. In this study, we found that rat corneal endothelial cells could spontaneously undergo EnMT during ex vivo culture. This change in rat corneal endothelial cells was associated with Notch signaling pathway activation after the first passage, which was blocked by the Notch inhibitor $N$-[N-(3,5-Difluorophenacetyl)-L-alanyl]-S-phenylglycine $t$-butyl ester (DAPT). This inhibitor also prevented transforming growth factor $\beta 1-, \beta 2-$, and $\beta 3$-induced EnMT and reversed transformed rat corneal endothelial cells to a normal phenotype. Furthermore, DAPT treatment blocked retrocorneal membrane formation in a rat corneal endothelium damage model. Our study indicates that the Notch signaling pathway is involved in the corneal EnMT process, which may be a novel therapeutic target for treating corneal endothelial fibrogenic disorders. (Am J Pathol 2013, 183: 786-795; http:// dx.doi.org/10.1016/j.ajpath.2013.05.025)
\end{abstract}

The corneal endothelium is a monolayer of uniform-sized hexagonal cells with low-resistance tight junctions that serves as a boundary between the stroma and anterior chamber. The maintenance of corneal endothelial normal cell morphological characteristics with intact tight junctions and cell density is critical for preserving corneal hydration, transparency, and, thus, visual acuity. ${ }^{1,2}$ Adult human corneal endothelial cells (CECs) are mitotically inactive, at least partly because of lack of mitogenic stimulation, ${ }^{3}$ cell contact inhibition, and the presence of anti-mitogenic factors in the aqueous humor. ${ }^{4,5}$

When the corneal endothelium is injured, the nearby normal CECs enlarge and migrate to fill the space resulting from loss of injured cells. ${ }^{6,7}$ However, there is emerging evidence suggesting that CECs can undergo endotheliumto-mesenchymal transition (EnMT) after chemical, mechanical, or other injury. ${ }^{8-11}$ During this transformation, quiescent endothelial cells become responsive to mitogens. This change encompasses altered cell morphological characteristics and collagen pattern expression associated with increases in $\alpha$-smooth muscle actin ( $\alpha$-SMA) expression, resulting from myofibroblast formation. In addition, downregulation of $\mathrm{N}$-cadherin expression occurs, along with a switch of type IV-rich basement membrane collagen synthesis to type I-rich fibrillar collagen synthesis, resulting in an abnormal fibrillar extracellular matrix. ${ }^{12-15}$ This change in collagen-type expression is indicated by the formation of a retrocorneal fibrous membrane (RCFM) between Descemet's membrane and the corneal endothelium, leading to corneal opacity and vision loss. ${ }^{11,16,17}$

Similar phenomena are also seen during ex vivo culture of CECs. Different methods have been used to expand CECs from various species. Although endothelial cells can proliferate, they can also easily lose their typical morphological characteristics and acquire a fibroblastic shape during

Supported by National Natural Science Foundation of China grants 81100639 (C.L.), U1205025 (Z.L.), 81270978 (Z.L.), 81000367 (N.D.), and 81070713 (W.L.), the Fundamental Research Funds for the Central Universities of China grants 2012121049 (W.L.) and 2011121063 (C.L.), and the Natural Science Foundation of Fujian Province of China grant 2010 D023 (N.D.).

C.L. and F.D. contributed equally to this work. 
expansion. ${ }^{18,19}$ In those cultures, fibroblast growth factor-2 was commonly used as a growth factor, which was shown to be a direct mediator of rabbit corneal EnMT. ${ }^{12,20-22}$ Therefore, protocols of sustaining CECs in culture may not be efficient. These considerations prompted us to determine whether EnMT inhibition may be beneficial for treating corneal endothelial fibrogenic disorders and promoting ex vivo expansion of CECs for tissue engineering.

Many studies have demonstrated that the activation of signaling pathways induced by transforming growth factor (TGF) $\beta$ and basic fibroblast growth factors could cause corneal EnMT. ${ }^{8,9,15,23-26}$ It was shown recently that the Notch signaling pathway is activated in regions that undergo EnMT during embryogenesis. ${ }^{27-29}$ In the present study, we used an ex vivo cell culture method and an in vivo endothelial wounding model to study the effects of Notch signaling on corneal EnMT. Our results indicate that the Notch signaling pathway is involved in CEC EnMT. This process is inhibited by a Notch signaling inhibitor, $N-[N-$ (3,5-difluorophenacetyl)-L-alanyl]-S-phenylglycine $\quad t$-butyl ester (DAPT), which is a $\gamma$-secretase inhibitor preventing Notch receptor proteolytic cleavage.

\section{Materials and Methods}

Human TGF- $\beta 1$, TGF- $\beta 2$, and TGF- $\beta 3$ recombinants, Dulbecco's modified Eagle's medium, Ham's F-12 medium, HEPES buffer, PBS, amphotericin B, gentamicin, fetal bovine serum, trypsin-EDTA, mouse epidermal growth factor, rabbit anti-zonula occludens protein (ZO)-1 and mouse anti-N-cadherin antibodies, and Alexa Fluor 546- or fluorescein isothiocyanate-conjugated donkey anti-mouse IgG were obtained from Invitrogen (Carlsbad, CA). Collagenase I was purchased from Roche (Indianapolis, IN). Hydrocortisone, dimethyl sulfoxide, cholera toxin, insulin-transferrin-sodium selenite media supplement, 3\% hydrogen peroxide, DAPI, MTT, Triton X-100, bovine serum albumin, DAPT, mouse anti- $\beta$-actin antibody, and protease and phosphatase inhibitor cocktails were from Sigma-Aldrich (St. Louis, MO). Mouse anti- $\alpha$-SMA and rabbit anti-Hes-1 antibodies were from Abcam (Cambridge, MA). Rabbit anti-Ki-67 and mouse anti-collagen IV antibodies were obtained from Santa Cruz Biotechnology (Santa Cruz, CA). The ExScript RT Reagent kit and SYBR Premix Ex Taq Kit were obtained from Takara Bio (Shiga, Japan).

\section{Rat Corneal Endothelial Cell Cultures}

Adult Sprague-Dawley rats were maintained and treated in accordance with the Association for Research in Vision and Ophthalmology Statement for the Use of Animals in Ophthalmic and Vision Research and according to an experimental procedure approved by the Committee for Animal Research at Xiamen University (Xiamen, Fujian, China). After the animal was sacrificed, the corneal tissues were harvested and endothelial cells with Descemet's membrane were mechanically separated from the stroma under a dissecting microscope, and then digested with $1 \mathrm{mg} /$ $\mathrm{mL}$ collagenase I in supplemented hormonal epithelial medium (SHEM; see later) for 16 hours. Rat corneal endothelial cell aggregates were dispersed into a cell suspension, and seeded into a 12-well plate and cultured in SHEM made of an equal volume of HEPES-buffered Dulbecco's modified Eagle's medium and Ham's F12. The medium was supplemented with $0.5 \%$ dimethyl sulfoxide, 2 $\mathrm{ng} / \mathrm{mL}$ mouse epidermal growth factor, $5 \mu \mathrm{g} / \mathrm{mL}$ insulin, 5 $\mu \mathrm{g} / \mathrm{mL}$ transferrin, $5 \mathrm{ng} / \mathrm{mL}$ selenium, $0.5 \mu \mathrm{g} / \mathrm{mL}$ hydrocortisone, $1 \mathrm{nmol} / \mathrm{L}$ cholera toxin, $5 \%$ fetal bovine serum, $50 \mu \mathrm{g} / \mathrm{mL}$ gentamicin, and $1.25 \mu \mathrm{g} / \mathrm{mL}$ amphotericin B. Some cells were cultivated in SHEM containing DAPT and/ or TGF- $\beta 1$, TGF- $\beta 2$, or TGF- $\beta 3$ at different concentrations. Cultures were incubated at $37^{\circ} \mathrm{C}$ in $5 \% \mathrm{CO}_{2}$, and the medium was changed every 2 days. Cells were passaged when they reached $80 \%$ confluence until the fifth passage.

\section{Cell Proliferation Assay}

To determine the growth curve, the fifth passage of CECs $\left(1.2 \times 10^{4}\right.$ cells per well $)$ was seeded onto 24 -well plates, with or without $10 \mu \mathrm{mol} / \mathrm{L}$ DAPT. Cells were harvested from three randomly selected wells by trypsin digestion and counted using a hemocytometer from day 1 to day 7. Cells from each well were counted three times, and the average cell concentration was used to construct the growth curve.

The effect of DAPT on cell proliferation was also measured by the MTT assay. The fifth passage of CECs was plated at a density of $3 \times 10^{3}$ cells per well in 96-well plates overnight and then treated with different concentrations of DAPT $(0,2.5$, $5,7.5,10,12.5,15$, and $17.5 \mu \mathrm{mol} / \mathrm{L}$ ). The final concentrations of dimethyl sulfoxide (DMSO) were $<0.1 \%$. After incubation for 24,72 , and 120 hours at $37^{\circ} \mathrm{C}$ in a humidified incubator, 5 $\mathrm{mg} / \mathrm{mL}$ MTT reagent in PBS was added to each well and incubated for 4 hours; after that, the medium was removed and $0.1 \mathrm{~mL}$ of buffered DMSO was added to each well. The absorbance was measured spectrophotometrically at $570 \mathrm{~nm}$ with a Bio-Tek ELX800 microplate reader (Bio-Tek Instruments, Winooski, VT). The effect of DAPT on cell proliferation was assessed as the percentage of inhibition, by which vehicle-treated cells at each time point were taken as control.

\section{Rat Corneal Endothelium Damage Model}

The rat corneal endothelium damage procedure was performed, as described previously, with modification. ${ }^{30}$ In brief, 12-week-old Sprague-Dawley rats were anesthetized with 60 $\mathrm{mg} / \mathrm{kg}$ i.p. ketamine and topical proparacaine solution to the cornea. A 3-mm-diameter stainless steel probe, cooled with liquid nitrogen, was applied once a day to the central cornea of the right eye of the rats $(n=12)$ for 30 seconds for 4 consecutive days. Both $0.1 \%$ atropine and $0.3 \%$ gentamicin sulfate were instilled in each eye after the procedure. 
Table 1 Primer Sequence Pairs Used for Quantitative Real-Time PCR

\begin{tabular}{|c|c|c|c|}
\hline Gene name & Gene symbol & Sense & Antisense \\
\hline$\beta$-Actin & ACTB & 5'-GCTATTTGGCGCTGGACTT-3' & 5'-GCGGCTCGTAGCTCTTCTC-3' \\
\hline$\alpha-S M A$ & ACTA1 & 5'-ACTGAGCGTGGCTATTCCTT-3' & $5^{\prime}$-TTCTCCAGGGAAGAAGAGGA-3' \\
\hline VE-Cadherin & $\mathrm{CDH} 5$ & $5^{\prime}-$ TGAAGAACGAGGACAGCAAC - 3' & $5^{\prime}$-GAACTTGGTATGCTCCCGAT-3' \\
\hline ZO-1 & $T J P 1$ & $5^{\prime}$ - АCACCCACTCTTCCAGAACC- $3^{\prime}$ & $5^{\prime}$-AATTTGGCAGAACACCATCA-3' \\
\hline Cx43 & GJA1 & 5'-GCCTGATGACCTGGAGATTT-3' & $5^{\prime}$-TGAACACCCTAGGTGCATGT-3' \\
\hline Notch2 & NOTCH2 & $5^{\prime}$-TCCAAGGGACCTTGTCCTAC-3' & $5^{\prime}$-TAGAGGAGCGGAGTGTTCCT-3' \\
\hline Jagged1 & JAG1 & $5^{\prime}-$ AACCGGATGGAGTACATCGT-3' & $5^{\prime}$-AAGCCAGCTTGTCGAAACTT-3' \\
\hline Jagged2 & JAG2 & $5^{\prime}-$ GACATCAATCCCAACGACTG-3' & $5^{\prime}-$ TAGGCGTCACACTGGAACTC-3' \\
\hline Hes1 & HES1 & $5^{\prime}-$ GACACCGGACAAACCAAAG-3' & $5^{\prime}$-CGCCTCTTCTCCATGATAGG-3' \\
\hline
\end{tabular}

Afterward, $100 \mu \mathrm{L}$ DAPT (50 $\mu \mathrm{mol} / \mathrm{L}$ in $0.1 \%$ DMSO in PBS) or $0.1 \%$ DMSO alone was topically applied three times a day for 10 days. During the follow-up, corneas were observed with a slit-lamp microscope (Kanghua Science \& Technology Co, Ltd, Chongqing, China). The rat corneal thickness in different time points was measured with an ultrasound pachymeter (model SP-2000; Tomey, Nagoya, Japan). The animals were sacrificed 16 days after the initial freezing. Endothelial cells of some corneas were mechanically harvested for real-time PCR. Corneal parts were subjected to H\&E staining, immunostaining, and stereomicroscopy. Untreated rats served as controls for comparison.

\section{Histological Characteristics and Immunostaining}

Cultured rat CEC or corneal frozen sections were fixed in $4 \%$ paraformaldehyde for 20 minutes and prepared for immunostaining by rehydration in PBS, followed by incubation in
$0.2 \%$ Triton $\mathrm{X}-100$ for 10 minutes. After rinsing three times with PBS for 5 minutes each and pre-incubation with $2 \%$ bovine serum albumin to block non-specific staining, sections were incubated with anti- $\alpha$-SMA $(1: 50)$, Hes-1(1:100), or type IV collagen $(1: 100)$ primary antibodies overnight at $4{ }^{\circ} \mathrm{C}$. After washing with PBS three times for 10 minutes each, specimens were incubated with secondary donkey anti-mouse IgG Alexa Fluor 546 and fluorescein isothiocyanate conjugates at 1:500 for 45 minutes. After washing with PBS three additional times for 15 minutes, sections were counterstained with DAPI for 10 minutes and then mounted for analysis under a fluorescence microscope (Nikon, Tokyo, Japan).

\section{RNA Isolation and Real-Time PCR}

RNA was isolated using TRIzol (Invitrogen) and reverse transcribed to cDNA using the ExScript RT Reagent kit. Real-time PCR was performed with a StepOne Real-Time
A

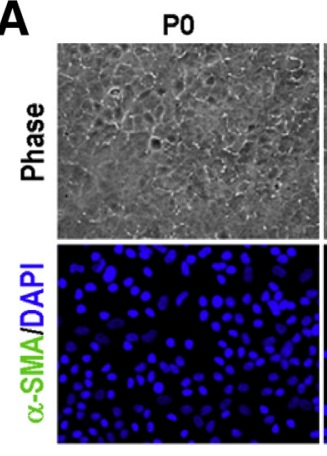

B

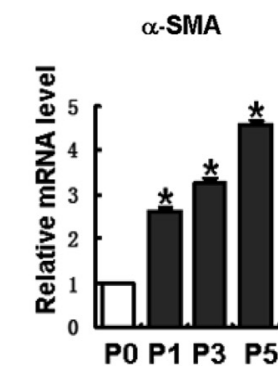

P1

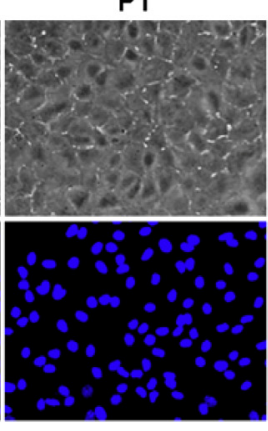

$C x-43$

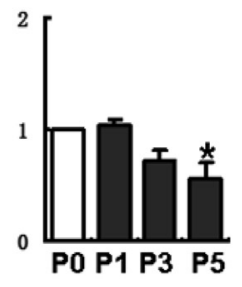

P3

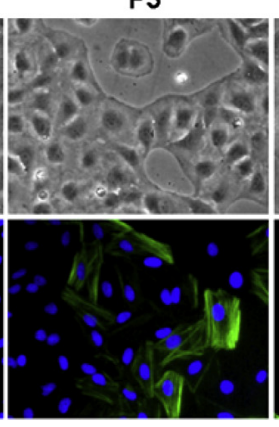

ZO-1

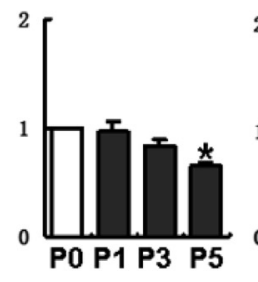

P5

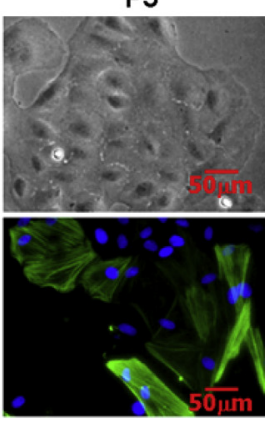

$\mathrm{N}$-Cadherin
Figure 1 Rat CECs exhibit spontaneous EnMT in vitro. The primary rat CECs have a uniform hexagonal shape in culture. However, the cell size gradually enlarged from passage 1 to passage 5 . Some cells turned into myofibroblastic-like cells from the second passage. A: Immunostaining of $\alpha$-SMA was undetectable in primary culture, whereas the number of $\alpha$-SMA-positive cells increased from passage 1 to passage 5. B: Realtime PCR of $\alpha$-SMA gene expression progressively increased from passage 1 to passage 5 , whereas $\mathrm{Cx} 43, \mathrm{ZO}-1$, and $\mathrm{N}$-cadherin gene expression gradually decreased. ${ }^{*} P<0.05$ by Student's $t$-test. 

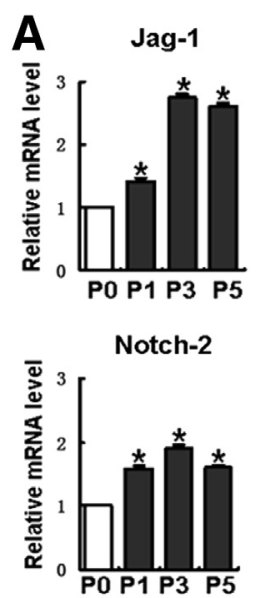

Jag-2

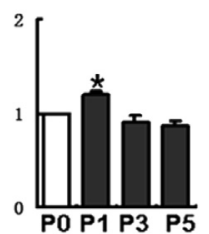

Hes-1

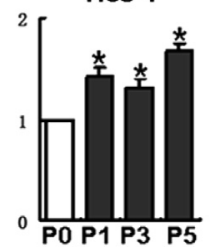

Notch-1

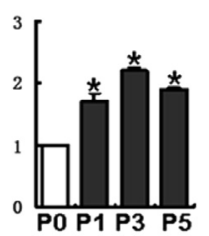

B

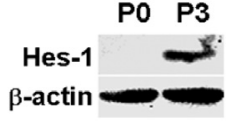

Figure 2 The Notch signaling pathway is activated during ex vivo culture of rat CECs. A: Jagged1 (Jag-1), Jag-2, Notch1, Notch2, and Hes1 mRNA levels significantly increased in P1 CECs, and all these genes, except for Jag-2, were maintained at high levels in P3 and P5 CECs. B: Western blot analysis of Hes1 was absent in P0 CECs, whereas there was strong expression in P3 CECs. ${ }^{*} P<0.05$ by Student's $t$-test.

PCR detection system (Applied Biosystems, Darmstadt, Germany) using an SYBR Premix Ex Taq Kit, according to the manufacturer's instructions. The amplification program included an initial denaturation step at $95^{\circ} \mathrm{C}$ for 10 minutes, followed by denaturation at $95^{\circ} \mathrm{C}$ for 10 seconds, and annealing and extension at $60^{\circ} \mathrm{C}$ for 30 seconds, for 40 cycles. SYBR Green fluorescence was measured after each extension step, and the specificity of amplification was evaluated by melting curve analysis. The primers used to amplify specific gene products from rat CEC cDNA are shown in Table 1. The results of the relative quantitative real-time PCR were analyzed by the comparative $\mathrm{C}_{\mathrm{T}}$ method and normalized to $\beta$-actin as an internal control.

\section{Western Blot Analysis}

CECs obtained after cultivation or corneal isolation were extracted in a cold lysis buffer composed of $50 \mathrm{mmol} / \mathrm{L}$ Tris- $\mathrm{HCl}(\mathrm{pH} 7.5), 150 \mathrm{mmol} / \mathrm{L} \mathrm{NaCl}, 1 \%$ Nonidet P-40, $0.5 \%$ sodium deoxycholate, $0.1 \%$ SDS, and protease and phosphatase inhibitor cocktails. Equal amounts of protein extracts $(20 \mu \mathrm{g})$ were subjected to Western blot analysis using $\beta$-actin $(1: 10,000)$, Hes-1 (1:100), $\alpha$-SMA (1:200), ZO-1 (1:100), N-cadherin (1:100), and Ki-67 (1:100) antibodies. The results were visualized by enhanced chemiluminescence reagents and recorded by Bio-Rad ChemiDoc XRS gel imaging system (Bio-Rad, Hercules, CA).

\section{Statistical Analysis}

All of the experiments were performed in triplicate and repeated at least three times each. Summary data are reported as means $\pm \mathrm{SD}$. Group means were compared using the appropriate version of the Student's unpaired $t$-test. Test results were considered significant if $P<0.05$.

\section{Results}

\section{Rat CECs Undergo in Vitro Spontaneous EnMT}

Rat CECs harvested from Descemet's membrane by collagenase digestion formed cell spheres (data not shown).
A

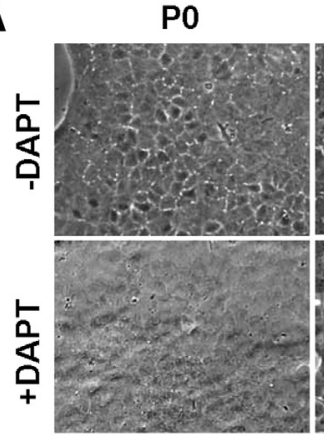

B

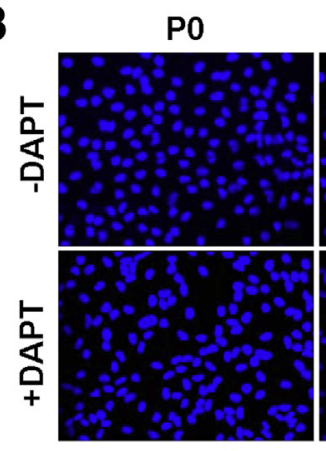

P1

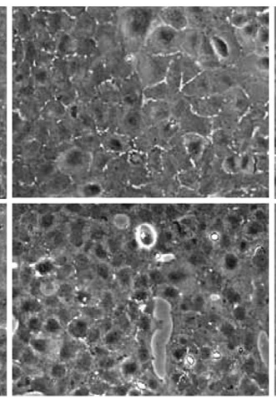

P1

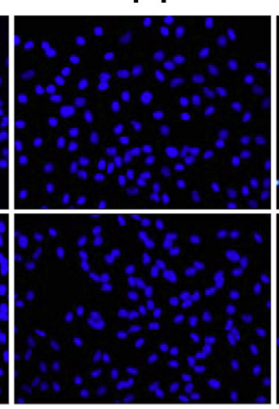

P3

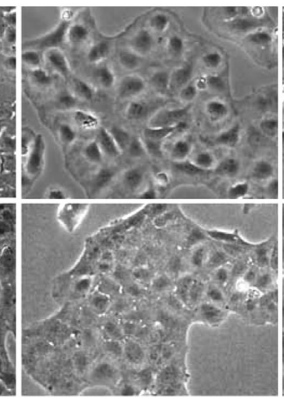

P3

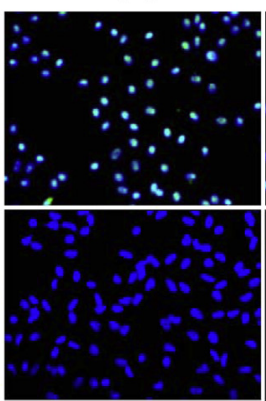

P5

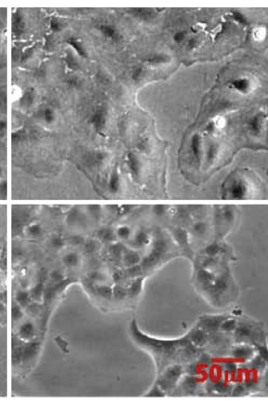

P5

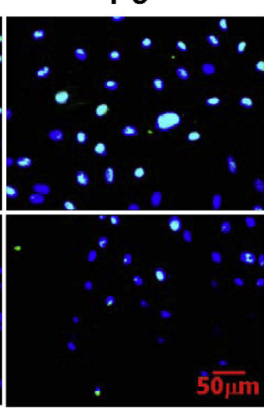

Hes1/DAPI

Figure 3 DAPT suppresses spontaneous EnMT of rat CECs in vitro. Phase-contrast microscopic images of rat CECs indicate changes in cell shape and increased cell size after serial passages in ex vivo culture without DAPT. A: CECs cultured with $10 \mu \mathrm{mol} / \mathrm{L}$ DAPT are more uniform in cell shape and smaller in cell size compared with the same passage of cells cultured without DAPT. B: Immunostaining shows strong expression of Hes1 in the third and fifth passage CECS without DAPT, whereas Hes1 is undectable from P0 to P3, and there are sporadic positive cells in P5 CECs. 

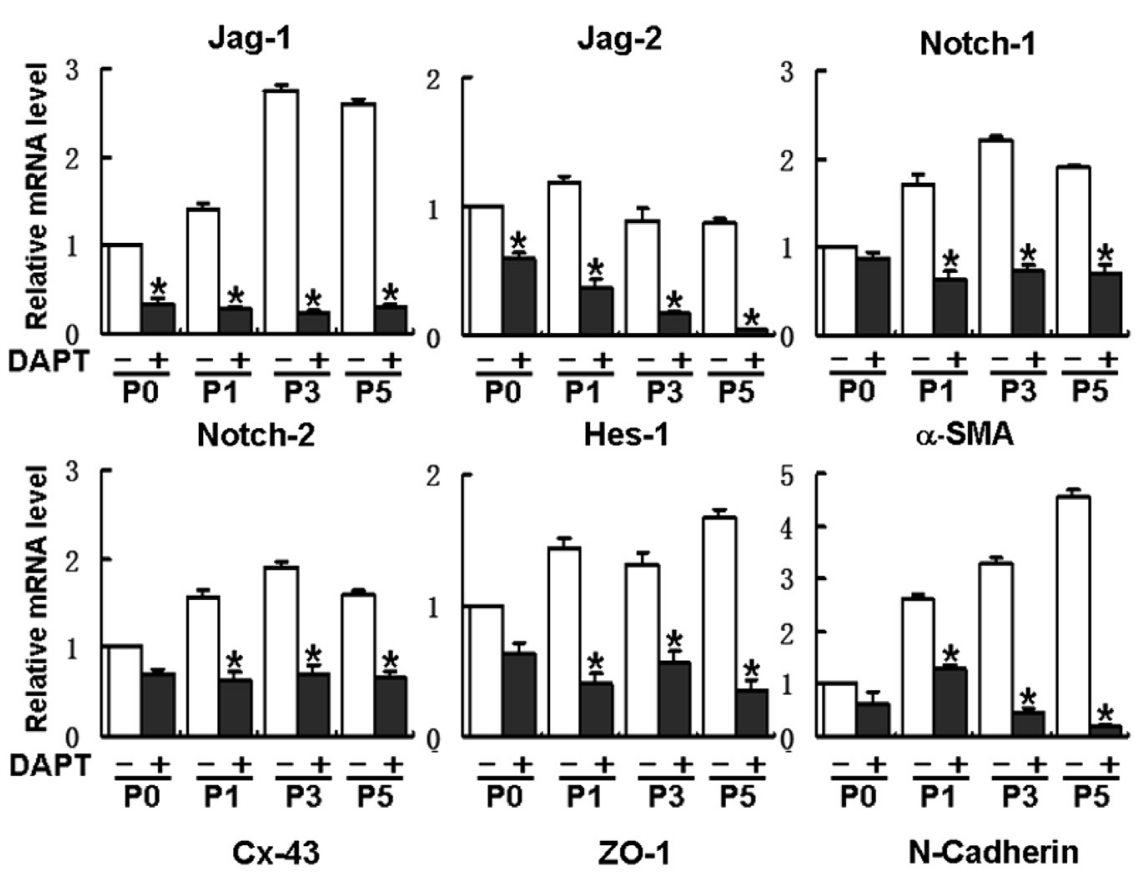

Figure 4 DAPT suppresses EnMT-related gene expression in rat CECs. Quantitative real-time PCR showed significant down-regulation of Jagged1 (Jag-1), Jag-2, Notch1, Notch2, Hes1, and $\alpha$-SMA gene expression, whereas there is up-regulation of $\mathrm{C} \times 43, \mathrm{ZO}-1$, and $\mathrm{N}$-cadherin gene expression from $\mathrm{P} 1$ to $\mathrm{P} 5$ in rat CECs cultured with $10 \mu \mathrm{mol} / \mathrm{L}$ DAPT, compared with those cultured without DAPT. ${ }^{*} P<$ 0.05 by Student's $t$-test.

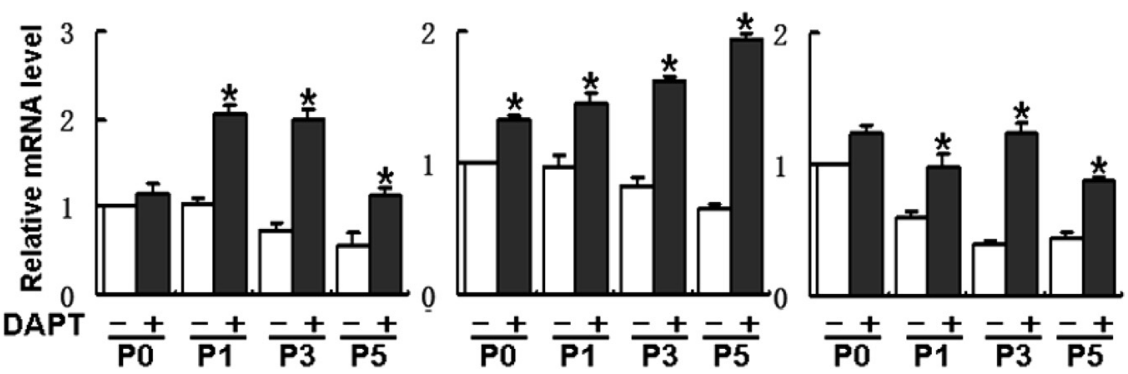

This response is similar to that described for human CECs. ${ }^{31}$ After seeding on plastic dishes, they attached and spread out in 1 day. The CECs had a uniform hexagonal shape after the first passage. Some CECs began to change their shape by appearing myofibroblast-like in the second passage, and underwent enlargement after being passed several more times. The number of $\alpha$-SMA-positive cells increased from passage 1 to passage 5 (Figure 1A). Realtime PCR showed that this change was associated with stepwise increases in $\alpha$-SMA gene expression, whereas cell junction marker gene expression of connexin 43 (Cx43), ZO-1, and N-cadherin instead gradually decreased (Figure 1B). These results indicate that some rat CECs progressively differentiated into a mesenchymal phenotype during ex vivo culture.

\section{Notch Signaling Pathway Mediates EnMT}

To determine whether the Notch signaling pathway is involved in corneal EnMT during ex vivo culture, real-time PCR was performed to detect Notch signal-related genes. Notch signaling ligands, Jagged1, Jagged2, Notch1, and Notch2, and downstream transcription factor Hes-1 gene expression increased in passage $(\mathrm{P}) 1 \mathrm{CECs}$, and all of the genes, except for Jagged2, remained high at P3 and P5 (Figure 2A). Western blot analysis confirmed prominent expression of Hes 1 in the third passage CECs (Figure 2B).

To validate Notch signaling pathway involvement in EnMT, the effect was evaluated of a Notch signaling pathway inhibitor, DAPT $(10 \mu \mathrm{mol} / \mathrm{L})$, on this transition. DAPT prevented the previously mentioned phenotypic CEC changes between passages 1 and 5. The cells remained uniformly hexagonal and relatively smaller than the same passage of cells cultured without DAPT (Figure 3A). Immunostaining of Hes-1 was undetectable in P0 and P1, whereas there was strong nuclear Hes-1 staining in P3 and P5 in serial cultures without DAPT. However, in serial cultures with DAPT, Hes-1 staining remained negative in passage 3 , and there were only a few positive cells in passage 5 (Figure 3B). Quantitative realtime PCR showed that Jagged1, Jagged2, Notch1, Notch2, Hes1, and $\alpha$-SMA were down-regulated, whereas $\mathrm{Cx} 43$, ZO-1, and N-cadherin gene expression levels were upregulated from P1 to P5 compared with CEC cultured without DAPT (Figure 4). Collectively, these results indicate that DAPT inhibited increases in Notch signaling pathway gene and protein expression during spontaneous EnMT of rat CECs. 


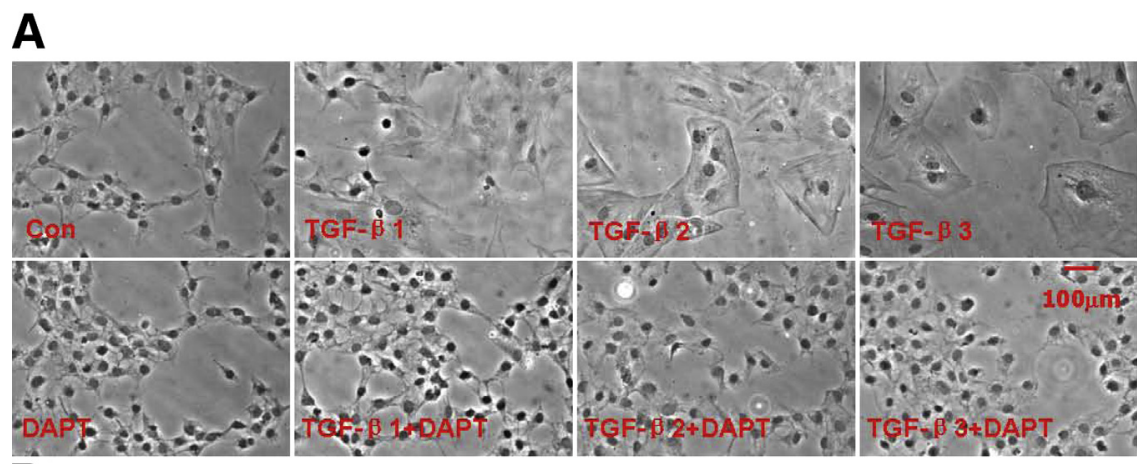

\section{B}

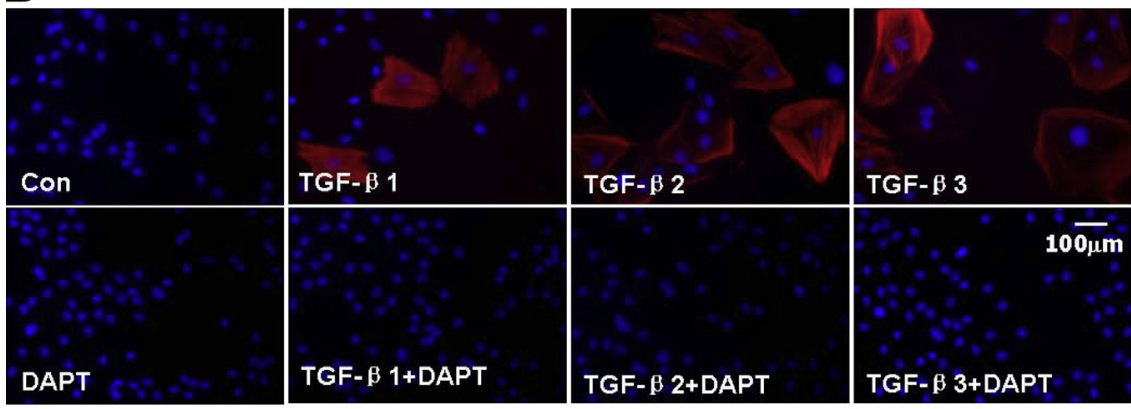

C

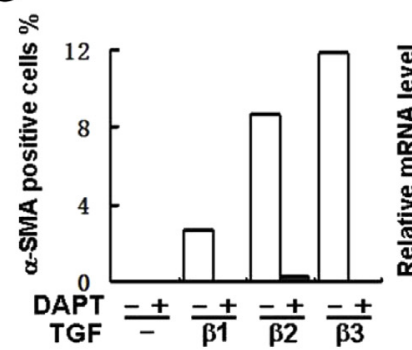

ZO-1

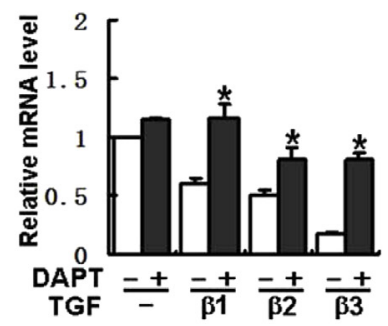

$\alpha$-SMAVDAPI

$\alpha$-SMA

Cx-43

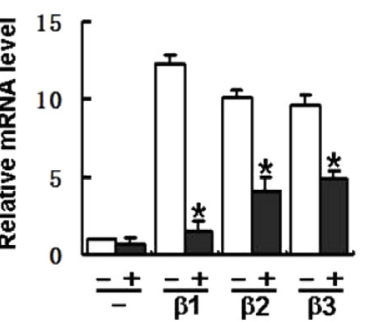

$\mathrm{N}-$ Cadherin

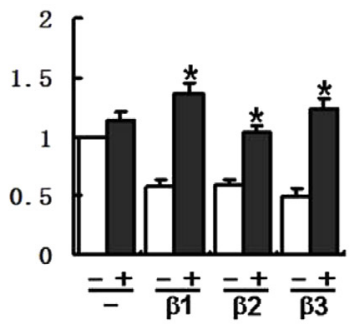

Figure 5 DAPT suppresses EnMT of rat CECS induced by TGF- $\beta$. TGF- $\beta 1-$, TGF- $\beta 2-$, and TGF- $\beta 3-$ treated CECs became elongated with filopodia. Many of them lost contact with their neighbors. A: In contrast, DAPT-treated CECs maintain a typical hexagonal appearance in the presence of TGF- $\beta$ s. B: Immunofluorescence staining shows many $\alpha$-SMApositive cells in TGF- $\beta$-treated P1 cells but not in the cultures with DAPT. C: Cell counting shows a significant difference between number of $\alpha$-SMApositive cells in cultures with or without DAPT. Realtime PCR shows DAPT reverses changes in $\alpha$-SMA, $\mathrm{Cx} 43, \mathrm{ZO}-1, \mathrm{~N}$-cadherin, and VE-cadherin gene expression induced by exposure to each of the three different TGF- $\beta$ isoforms. Con, control. ${ }^{*} P<0.05$ by Student's $t$-test.

\section{Notch Inhibitor Suppresses TGF- $\beta$-Induced EnMT}

Because Notch signaling is one of the pathways mediating TGF- $\beta$-induced EnMT in other tissues, ${ }^{32}$ we determined if it mediates this response to TGF- $\beta 1$ in rat CEC. To make this assessment, we compared the effects of TGF- $\beta 1$, TGF$\beta 2$, or TGF- $\beta 3$ on second passage CECs in the presence and absence of DAPT. As indicated, the TGF- $\beta 1 / 2 / 3$-treated cells became elongated after 24 hours, and many of them lost contact with their neighbors. By 48 hours, most of the cells exhibited a fibroblastic spindle-like shape. In contrast, the DAPT-treated CECs maintained their attachments to each other and had a typical hexagonal appearance (Figure 5A). After exposure to TGF- $\beta 1 / 2 / 3$ for 3 days, approximately $3 \%$ to $12 \%$ of the CECs underwent EnMT based on appearance of anti- $\alpha$-SMA antibody staining. TGF- $\beta 3$ was most potent in inducing EnMT (Figure 5C). However, simultaneous exposure to DAPT almost completely blocked this response (Figure 5B). TGF- $\beta 1 / 2 /$ 3 -induced effects were determined on $\alpha$-SMA, Cx43, ZO$1, \mathrm{~N}$-cadherin, and VE-cadherin gene expression in the presence and absence of DAPT. Real-time PCR results showed that $\alpha$-SMA and VE-cadherin expression levels were up-regulated by TGF- $\beta 1 / 2 / 3$, which was blocked by 

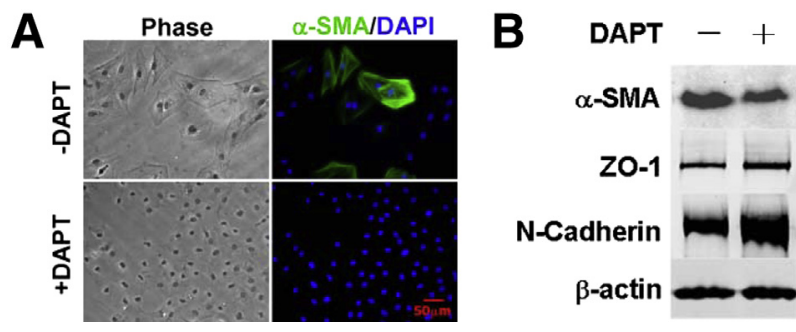

Figure 6 DAPT reverses EnMT phenotype of rat CECs in vitro. Phasecontrast microscopy shows that fifth passage rat CECS have a fibroblasticlike appearance and are strongly stained for $\alpha$-SMA. A: After being cultured for 72 hours with $10 \mu \mathrm{mol} / \mathrm{L}$ DAPT, the cells revert back to a hexagonal shape, and $\alpha$-SMA expression dramatically decreases. B: Western blot results show $\alpha$-SMA protein expression undergoes downregulation, whereas $\mathrm{ZO}-1$ and $\mathrm{N}$-cadherin expression increases in fifth passage CECs cultured with DAPT for 72 hours.

DAPT. On the other hand, the cell junction gene markers ZO-1 and N-cadherin and the gap junction gene marker Cx43 were down-regulated by TGF- $\beta 1 / 2 / 3$, whereas DAPT addition reversed these declines (Figure 5C).

\section{Notch Inhibitor Reverses in Vitro EnMT}

To determine whether EnMT can be reversed by Notch signaling pathway blockage, fifth passage rat CECs were cultured with or without $10 \mu \mathrm{mol} / \mathrm{L}$ DAPT for 72 hours. Without DAPT, they were spindle shaped, with prominent filopodia. However, with $10 \mu \mathrm{mol} / \mathrm{L}$ DAPT, the cells remained hexagonal without filopodia. Similarly, $\alpha$-SMA was more highly expressed in passage 5 , whereas with 10 $\mu \mathrm{mol} / \mathrm{L}$ DAPT, there were only a sparse number of $\alpha$ SMA-positive cells (Figure 6A). Again, $\alpha$-SMA expression was down-regulated, whereas ZO-1 and $\mathrm{N}$-cadherin were up-regulated, in the fifth passage of CECs treated with DAPT (Figure 6B). Taken together, these results indicate that Notch signaling pathway inhibition could cause CECs to regain their parental phenotype.

\section{Notch Inhibitor Reduces Proliferation and Migration of Transformed CECs}

To further evaluate the effect of DAPT on CEC proliferation, we performed a cell growth assay in parallel with Ki-67 immunostaining. DAPT $(10 \mu \mathrm{mol})$ slightly reduced CEC proliferation from days 3 to 7 by up to approximately $13 \%$ compared with the vehicle-treated control (Figure 7A). These declines are consistent with Ki-67 down-regulation (Figure 7B). We further investigated the dose-dependent effects of DAPT as a function of time on CEC proliferation using the MTT assay. Only after 120 hours of exposure to any of the concentrations from 5 to $17.5 \mu \mathrm{mol} / \mathrm{L}$ did DAPT suppress CEC proliferation. With $10 \mu \mathrm{mol} / \mathrm{L}$ DAPT at day 5 , proliferation decreased by approximately 18\% (Figure 7C). Figure 7D shows that cell migration at 48 hours was inhibited by $10 \mu \mathrm{mol} / \mathrm{L}$ DAPT based on the increase in the remaining wound compared with the vehicle-treated control. These results indicate that inhibition of the Notch signaling pathway could reduce CEC proliferation and migration.

\section{Notch Inhibitor Prevents Injury-Induced Rat} Retrocorneal Fibrous Membrane Formation

To assess whether the Notch pathway can be a therapeutic target to suppress RCFM formation, a rat corneal endothelial wound-healing model was used. The effect was determined of topical DAPT application on a transcorneal wound caused by a cryogenic probe. Slit-lamp and stereomicroscopic examination after 16 days showed that the stroma became edematous and transparency declined. H\&E staining shows that RCFM formation occurred based on the appearance of fibrous tissue containing spindle-shaped fibroblast-like cells on the posterior corneal surface.

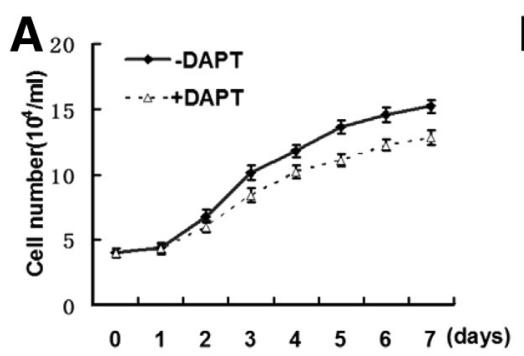

B

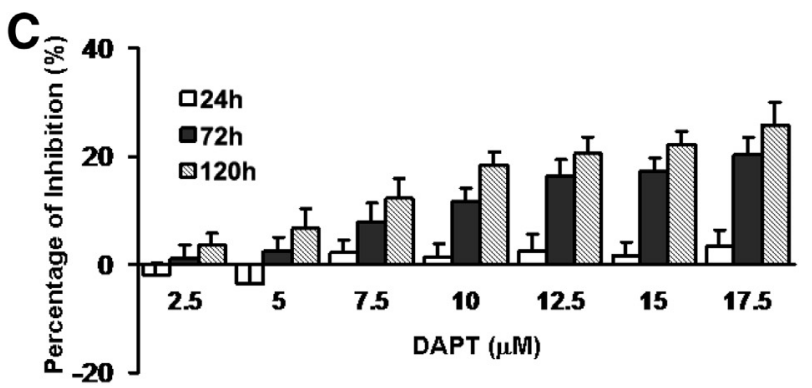

D

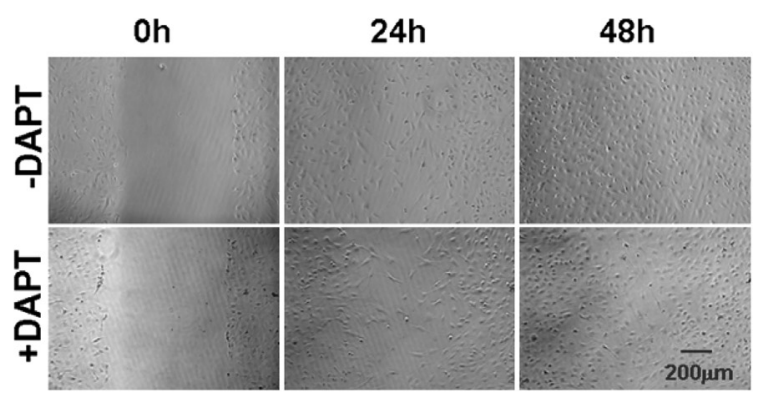

Figure 7 DAPT reduces proliferation and migration of rat CECs containing a mesenchymal phenotype. A: The dose- and time-dependent effects of DAPT on proliferation were determined on fifth passage rat CECs. Cell number is lower in CECs treated with $10 \mu \mathrm{mol} / \mathrm{L}$ DAPT from day 3 to day 7 than in its absence $(P<0.05)$. B: Western blots of Ki-67 indicate that CEC proliferation declines after 7 days of exposure to $10 \mu \mathrm{mol} / \mathrm{L}$ DAPT. C: The effects of DAPT from 2.5 to $17.5 \mu \mathrm{mol} / \mathrm{L}$ on CEC proliferation, as a function of exposure time from 24 to 120 hours, show that $5 \mu \mathrm{mol} / \mathrm{L}$ DAPT begins to suppress proliferation only after 72 and 120 hours. Progressive increases in DAPT concentration up to $17.5 \mu \mathrm{mol} / \mathrm{L}$ progressively reduce proliferation. D: A scratch cell migratory wound-healing assay shows 10 $\mu \mathrm{mol} / \mathrm{L}$ DAPT inhibited cell migration compared with untreated passagematched control cells. 


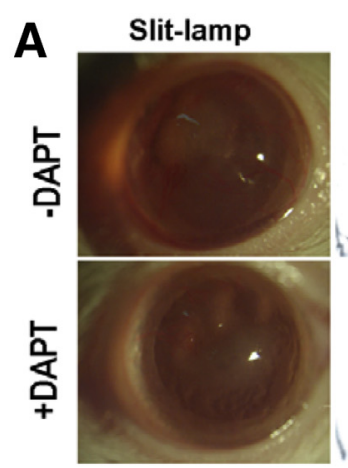

B

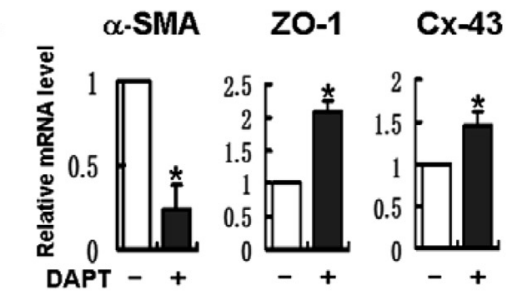

N-Cadherin Ki67 VE-Cadherin
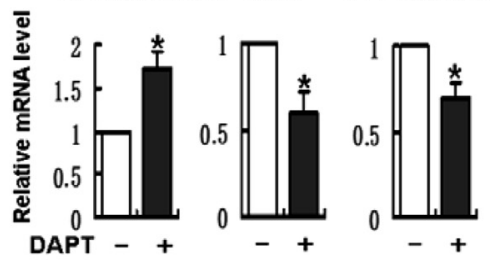

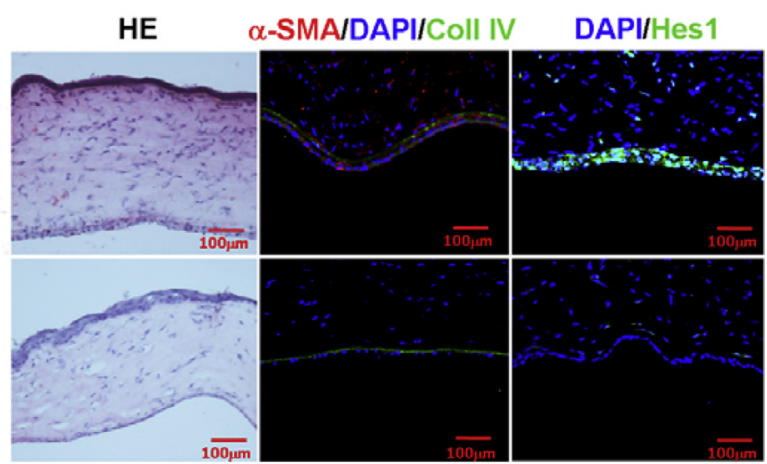

C

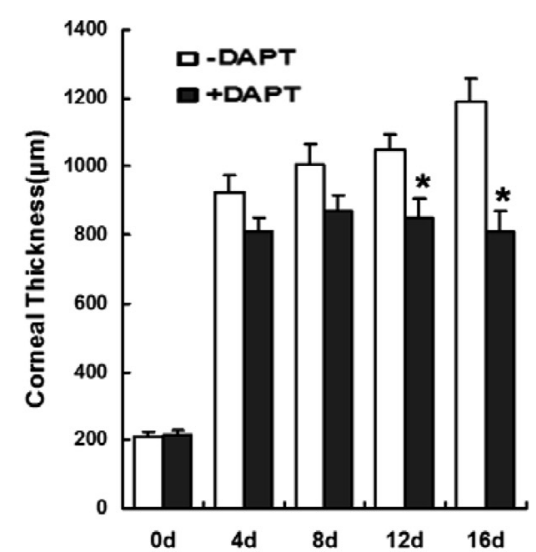

Figure 8 DAPT inhibits RCFM formation. At 2 weeks after cyroprobe wounding, the rat cornea became stromal edematous and less transparent, based on slit-lamp microscopy and stereomicroscopic examinations. H\&E staining reveals RCFM formation in the wounded eyes, characterized by fibrous tissue containing fibroblast-like cells on the posterior corneal surface. A: Collagen (Coll) IV and $\alpha$-SMA double immunostaining demonstrates $\alpha$-SMA-positive cells posterior to the Descemet's membrane and $\alpha$-SMA-positive cells in the injured stroma. Hes 1 immunostaining is evident in the cells in RCFM and some cells in corneal stroma. In DAPT-treated rat corneas, slit-lamp microscopy reveals mild corneal edema, and stereomicroscopy show that rat corneas were more transparent than the untreated controls. Furthermore, after $10 \mu \mathrm{mol} / \mathrm{L}$ DAPT treatment, H\&E staining shows that the stromal layer is thinner, with less cellularity, and there is no fibrous tissue present in the posterior corneal surface. Collagen IV and $\alpha$-SMA double staining in the endothelial monolayer does not reveal any $\alpha$-SMA-staining cells either posterior to the Descemet's membrane or in the stroma. Similarly, in this layer, there are no Hes1-positive immunostaining cells. A few positive cells are present in the posterior corneal stroma. B: Real-time PCR show that $\alpha$-SMA, Ki-67, and VE-cadherin gene expression levels were lower, whereas $\mathrm{Z0}-1, \mathrm{C} \times 43$, and $\mathrm{N}$-cadherin gene expression levels are higher, in wounded endothelium with DAPT treatment. C: Ultrasound pachymetry reveals that corneal thickness is significantly thinner on days 12 and 16 in DAPT-treated corneas. ${ }^{*} P<0.05$ by Student's $t$-test.

Collagen IV and $\alpha$-SMA double staining confirmed that most of these cells were $\alpha$-SMA positive, and situated posterior to Descemet's membrane. There were also $\alpha$ SMA-positive cells present in the injured corneal stroma. Hes1 staining was evident in most of the cells that composed RCFM and in some stromal cells. In contrast, after topical application of DAPT for 16 days, there was less stromal edema and the dissected cornea remained more transparent. The thinner stroma had a lower cell density. Moreover, there was no fibrous tissue present on the posterior corneal surface. Collagen IV and $\alpha$-SMA double staining showed that the endothelial monolayer posterior to Descemet's membrane was $\alpha$-SMA negative, and there were no $\alpha$-SMA-positive cells in the stroma. Furthermore, there were no Hes1-positive cells in the corneal endothelial layer and much fewer positive cells in the posterior corneal stroma (Figure 8A). Real-time PCR evaluated Descemet's membrane and endothelial $\alpha$-SMA, Ki-67, ZO-1, Cx43, $\mathrm{N}$-cadherin, Ki-67, and VE-cadherin gene expression.
DAPT inhibited mRNA levels of $\alpha$-SMA, Ki-67, and VEcadherin, whereas ZO-1, Cx43, and N-cadherin levels increased (Figure 8B). Ultrasound pachymetry revealed that the cornea was thinner in the DAPT-treated group compared with the control group after 12 days of DAPT treatment (Figure 8C). These results demonstrate that DAPT blocked RCFM formation and reduced declines in tissue transparency resulting from corneal cryoinjury.

\section{Discussion}

The Notch signaling pathway is evolutionarily conserved and plays a critical role in embryonic development. It serves as a regulator of short-distance intercellular communication for cell fate determination and maintenance of stem cells. ${ }^{33}$ Moreover, Notch signaling is involved in both morphogenesis during development and many pathogenic processes. Aberrant Notch signaling can induce tumorigenesis in different organs, such as the mammary gland, ${ }^{34}$ intestine, ${ }^{35}$ and skin. ${ }^{36}$ Notch 
signaling is also activated during the healing of injured tissues, such as $\operatorname{skin}^{37}$ and corneal epithelium. ${ }^{38}$

Recently, it has become apparent that Notch signaling is also involved in controlling EnMT. For example, Notch signaling-induced EnMT is required in early cardiac valve formation during development. ${ }^{39,40}$ Jagged1-Notch interactions induce EnMT of microvascular endothelial cells. ${ }^{27}$ In the present study, we demonstrated, for the first time to our knowledge, that the Notch signaling pathway is involved in EnMT of CECs. Notch signaling activation started from the first passage, as evidenced by Notch-related gene expression of Jagged1, Jagged2, Notch1, Notch2, and Hes1, and became prominent in the later passages of cultured CECs (Figure 2). Emergence of $\alpha$-SMA expression, a hallmark of EnMT, was synchronous with Hes-1 expression, indicating an association between EnMT and Notch signaling pathway activation. Our observation that addition of a $\gamma$-secretase inhibitor, DAPT, to the medium preserved the corneal endothelial cell phenotype (Figures 3 and 4) lends support to the notion that the Notch signaling pathway plays a crucial role in EnMT of CECs.

In our study, DAPT almost completely blocked EnMT induced by TGF- $\beta$, supporting the notion that Notch signaling is downstream of TGF- $\beta$ signaling. TGF- $\beta$-induced signaling plays a key role in CEC EnMT and the development of posterior RCFM formation. ${ }^{25}$ Inhibition of this pathway prevents injury-induced corneal endothelial fibrosis. ${ }^{10}$ These results are in agreement with our finding that TGF- $\beta 1$,TGF$\beta 2$, and TGF- $\beta 3$ induced EnMT of rat CECs. Interestingly, the addition of DAPT significantly reduced TGF- $\beta$-induced EnMT (Figure 5). Other studies in various cell types identified Notch and TGF- $\beta$ receptor-induced signaling, eliciting a variety of responses. For example, TGF- $\beta 1$ induces Notch signaling activation in primary rat peritoneal mesothelial cells. DAPT blocks this activation and prevents peritoneal fibrosis through inhibiting epithelial-to-mesenchymal transition. ${ }^{41}$ Activation of TGF- $\beta$ signaling can also up-regulate Hes-1 expression in myogenic cells. ${ }^{42}$ On the other hand, Notch activation by TGF- $\beta$ also activates and increases R-Smad expression. These pathways control cardiac endothelial cell proliferation and differentiation. ${ }^{43}$ TGF- $\beta$ and Notch signaling pathways are integrated and coordinated to induce epithelialto-mesenchymal transitions in epithelial cells of lung alveoli, ${ }^{44}$ kidney tubules ${ }^{45}$ and epidermis. ${ }^{46}$ Our data suggest that cross talk between TGF- $\beta$ and Notch signaling pathways also has a role in EnMT control. Similarly, in embryonic heart development, Notch signaling promotes TGF- $\beta$-mediated EnMT that leads to cellularization of developing cardiac valvular primordia. ${ }^{47}$

Transformed endothelial cells normally exhibit higher proliferation and migration activity than the parental CECs. On the other hand, we found that DAPT application reduced the CEC proliferation rate, and slightly inhibited endothelial migration. This finding agrees with a study showing that $\gamma$ secretase inhibitor had similar effects in a corneal epithelial cell culture, whereas Notch activation by Jagged 1 stimulated corneal epithelial cell proliferation. ${ }^{48}$ One may propose that inhibition of the Notch signaling pathway may have a negative effect on corneal endothelial wound healing. However, our in vivo study revealed that topical DAPT application after freeze injury significantly reduced corneal edema and improved transparency. These effects could be a consequence of inhibition of EnMT by DAPT, resulting in a higher density of CEC and improved corneal endothelial function restoration, which is needed to offset the natural tendency of the stroma to swell and become less transparent. RCFM formation decreased, to a large extent, after DAPT treatment (Figure 8), along with fewer stromal $\alpha$-SMA- and Hes1 -positive cells. In contrast, without DAPT exposure, these cell types were in abundance. This difference indicates Notch activation is involved in RCFM formation and in the stromal wound-healing process accompanied by fibrosis. Notch signaling also contributes to corneal epithelial wound healing based on its stimulation of cell proliferation and differentiation in a mouse wound-healing model. ${ }^{38}$

Because of a worldwide shortage of readily accessible donor corneal tissue for transplantation, the results are of potential clinical significance. Corneal availability may increase by inhibiting Notch signaling and reducing declines in CEC caused by EnMT. This approach may be used to also prevent RCFM formation in traumatized eyes. It was shown recently that i.p. injection of DAPT significantly attenuated peritoneal fibrosis in a rat model. ${ }^{41}$ Such a procedure could also be used to maintain and expand corneal endothelial cells in culture without losing them through EnMT. Other studies are being planned to determine the benefit of this Notch inhibitor in human endothelial tissue engineering. The inhibition by DAPT of fibrosis in a rat corneal endothelial injury is being evaluated by us as a treatment of corneal endothelial fibrogenic disorders in humans. This study may shed new light on how to develop effective regimens for treatment of EnMT-related fibrosis diseases.

\section{References}

1. Kaye GI, Sibley RC, Hoefle FB: Recent studies on the nature and function of the corneal endothelial barrier. Exp Eye Res 1973, 15: 585-613

2. Waring GO 3rd, Bourne WM, Edelhauser HF, Kenyon KR: The corneal endothelium: normal and pathologic structure and function. Ophthalmology 1982, 89:531-590

3. Welge-Lussen U, May CA, Neubauer AS, Priglinger S: Role of tissue growth factors in aqueous humor homeostasis. Curr Opin Ophthalmol 2001, 12:94-99

4. Joyce NC, Harris DL, Mello DM: Mechanisms of mitotic inhibition in corneal endothelium: contact inhibition and TGF-beta2. Invest Ophthalmol Vis Sci 2002, 43:2152-2159

5. Dalma-Weiszhauz J, Blumenkranz M, Hartzer M, Hernandez E: Intraocular extracellular cyclic nucleotide concentrations: the influence of vitreous surgery. Graefes Arch Clin Exp Ophthalmol 1993, 231:184-186

6. Sherrard ES: The corneal endothelium in vivo: its response to mild trauma. Exp Eye Res 1976, 22:347-357

7. Honda H, Ogita Y, Higuchi S, Kani K: Cell movements in a living mammalian tissue: long-term observation of individual cells in wounded corneal endothelia of cats. J Morphol 1982, 174:25-39 
8. Saika S, Yamanaka O, Okada Y, Tanaka S, Miyamoto T, Sumioka T, Kitano A, Shirai K, Ikeda K: TGF beta in fibroproliferative diseases in the eye. Front Biosci (Schol Ed) 2009, 1:376-390

9. Lee JG, Kay EP: NF-kappaB is the transcription factor for FGF-2 that causes endothelial mesenchymal transformation in cornea. Invest Ophthalmol Vis Sci 2012, 53:1530-1538

10. Sumioka T, Ikeda K, Okada Y, Yamanaka O, Kitano A, Saika S: Inhibitory effect of blocking TGF-beta/Smad signal on injury-induced fibrosis of corneal endothelium. Mol Vis 2008, 14:2272-2281

11. Nakano $Y$, Oyamada $M$, Dai $P$, Nakagami $T$, Kinoshita $S$, Takamatsu T: Connexin43 knockdown accelerates wound healing but inhibits mesenchymal transition after corneal endothelial injury in vivo. Invest Ophthalmol Vis Sci 2008, 49:93-104

12. Kay EP, Gu X, Ninomiya Y, Smith RE: Corneal endothelial modulation: a factor released by leukocytes induces basic fibroblast growth factor that modulates cell shape and collagen. Invest Ophthalmol Vis Sci 1993, 34:663-672

13. Hassell JR, Birk DE: The molecular basis of corneal transparency. Exp Eye Res 2010, 91:326-335

14. Leung EW, Rife L, Smith RE, Kay EP: Extracellular matrix components in retrocorneal fibrous membrane in comparison to corneal endothelium and Descemet's membrane. Mol Vis 2000, 6:15-23

15. Reneker LW, Bloch A, Xie L, Overbeek PA, Ash JD: Induction of corneal myofibroblasts by lens-derived transforming growth factor beta1 (TGFbeta1): a transgenic mouse model. Brain Res Bull 2010, 81:287-296

16. Michels RG, Kenyon KR, Maumence AE: Retrocorneal fibrous membrane. Invest Ophthalmol 1972, 11:822-831

17. Jakobiec FA, Bhat P: Retrocorneal membranes: a comparative immunohistochemical analysis of keratocytic, endothelial, and epithelial origins. Am J Ophthalmol 2010, 150:230-242.e2

18. Joyce NC, Zhu CC: Human corneal endothelial cell proliferation: potential for use in regenerative medicine. Cornea 2004, 23:S8-S19

19. Sumide T, Nishida K, Yamato M, Ide T, Hayashida Y, Watanabe K, Yang J, Kohno C, Kikuchi A, Maeda N, Watanabe H, Okano T, Tano Y: Functional human corneal endothelial cell sheets harvested from temperature-responsive culture surfaces. FASEB J 2006, 20:392-394

20. Kay EP, Gu X, Smith RE: Corneal endothelial modulation: bFGF as direct mediator and corneal endothelium modulation factor as inducer. Invest Ophthalmol Vis Sci 1994, 35:2427-2435

21. Gu X, Seong GJ, Lee YG, Kay EP: Fibroblast growth factor 2 uses distinct signaling pathways for cell proliferation and cell shape changes in corneal endothelial cells. Invest Ophthalmol Vis Sci 1996, 37: 2326-2334

22. Lee HT, Lee JG, Na M, Kay EP: FGF-2 induced by interleukin-1 beta through the action of phosphatidylinositol 3-kinase mediates endothelial mesenchymal transformation in corneal endothelial cells. J Biol Chem 2004, 279:32325-32332

23. Lee HT, Kay EP: FGF-2 induced reorganization and disruption of actin cytoskeleton through PI 3-kinase, Rho, and Cdc42 in corneal endothelial cells. Mol Vis 2003, 9:624-634

24. Lee JG, Kay EP: FGF-2-mediated signal transduction during endothelial mesenchymal transformation in corneal endothelial cells. Exp Eye Res 2006, 83:1309-1316

25. Petroll WM, Jester JV, Bean JJ, Cavanagh HD: Myofibroblast transformation of cat corneal endothelium by transforming growth factor-beta1, -beta2, and -beta3. Invest Ophthalmol Vis Sci 1998, 39:2018-2032

26. Ko MK, Kay EP: Regulatory role of FGF-2 on type I collagen expression during endothelial mesenchymal transformation. Invest Ophthalmol Vis Sci 2005, 46:4495-4503

27. Noseda M, McLean G, Niessen K, Chang L, Pollet I, Montpetit R, Shahidi R, Dorovini-Zis K, Li L, Beckstead B, Durand RE, Hoodless PA, Karsan A: Notch activation results in phenotypic and functional changes consistent with endothelial-to-mesenchymal transformation. Circ Res 2004, 94:910-917

28. Grego-Bessa J, Diez J, Timmerman L, de la Pompa JL: Notch and epithelial-mesenchyme transition in development and tumor progression: another turn of the screw. Cell Cycle 2004, 3:718-721
29. Leong KG, Niessen K, Kulic I, Raouf A, Eaves C, Pollet I, Karsan A: Jagged1-mediated Notch activation induces epithelial-to-mesenchymal transition through Slug-induced repression of E-cadherin. J Exp Med 2007, 204:2935-2948

30. Kay ED, Cheung CC, Jester JV, Nimni ME, Smith RE: Type I collagen and fibronectin synthesis by retrocorneal fibrous membrane. Invest Ophthalmol Vis Sci 1982, 22:200-212

31. Li W, Sabater AL, Chen YT, Hayashida Y, Chen SY, He H, Tseng SC: A novel method of isolation, preservation, and expansion of human corneal endothelial cells. Invest Ophthalmol Vis Sci 2007, 48: 614-620

32. van Meeteren LA, ten Dijke P: Regulation of endothelial cell plasticity by TGF-beta. Cell Tissue Res 2012, 347:177-186

33. Artavanis-Tsakonas S, Rand MD, Lake RJ: Notch signaling: cell fate control and signal integration in development. Science 1999, 284: 770-776

34. Han J, Hendzel MJ, Allalunis-Turner J: Notch signaling as a therapeutic target for breast cancer treatment? Breast Cancer Res 2012, 13:210

35. Guilmeau S: Notch signaling and intestinal cancer. Adv Exp Med Biol 2012, 727:272-288

36. Panelos J, Massi D: Emerging role of Notch signaling in epidermal differentiation and skin cancer. Cancer Biol Ther 2009, 8:1986-1993

37. Chigurupati S, Arumugam TV, Son TG, Lathia JD, Jameel S, Mughal MR, Tang SC, Jo DG, Camandola S, Giunta M, Rakova I, McDonnell N, Miele L, Mattson MP, Poosala S: Involvement of notch signaling in wound healing. PLoS One 2007, 2:e1167

38. Lu H, Lu Q, Zheng Y, Li Q: Notch signaling promotes the corneal epithelium wound healing. Mol Vis 2012, 18:403-411

39. Niessen K, Karsan A: Notch signaling in cardiac development. Circ Res 2008, 102:1169-1181

40. Chang AC, Fu Y, Garside VC, Niessen K, Chang L, Fuller M, Setiadi A, Smrz J, Kyle A, Minchinton A, Marra M, Hoodless PA, Karsan A: Notch initiates the endothelial-to-mesenchymal transition in the atrioventricular canal through autocrine activation of soluble guanylyl cyclase. Dev Cell 2011, 21:288-300

41. Zhu F, Li T, Qiu F, Fan J, Zhou Q, Ding X, Nie J, Yu X: Preventive effect of Notch signaling inhibition by a gamma-secretase inhibitor on peritoneal dialysis fluid-induced peritoneal fibrosis in rats. Am J Pathol 2010, 176:650-659

42. Blokzijl A, Dahlqvist C, Reissmann E, Falk A, Moliner A, Lendahl U, Ibanez CF: Cross-talk between the Notch and TGF-beta signaling pathways mediated by interaction of the Notch intracellular domain with Smad3. J Cell Biol 2003, 163:723-728

43. Fu Y, Chang A, Chang L, Niessen K, Eapen S, Setiadi A, Karsan A: Differential regulation of transforming growth factor beta signaling pathways by Notch in human endothelial cells. J Biol Chem 2009, 284: 19452-19462

44. Aoyagi-Ikeda K, Maeno T, Matsui H, Ueno M, Hara K, Aoki Y, Aoki F, Shimizu T, Doi H, Kawai-Kowase K, Iso T, Suga T, Arai M, Kurabayashi M: Notch induces myofibroblast differentiation of alveolar epithelial cells via transforming growth factor-\{beta\}-Smad3 pathway. Am J Respir Cell Mol Biol 2011, 45:136-144

45. Nyhan KC, Faherty N, Murray G, Cooey LB, Godson C, Crean JK, Brazil DP: Jagged/Notch signalling is required for a subset of TGFbeta1 responses in human kidney epithelial cells. Biochim Biophys Acta 2010, 1803:1386-1395

46. Zavadil J, Cermak L, Soto-Nieves N, Bottinger EP: Integration of TGF-beta/Smad and Jagged1/Notch signalling in epithelial-tomesenchymal transition. EMBO J 2004, 23:1155-1165

47. Timmerman LA, Grego-Bessa J, Raya A, Bertran E, Perez-Pomares JM, Diez J, Aranda S, Palomo S, McCormick F, Izpisua-Belmonte JC, de la Pompa JL: Notch promotes epithelial-mesenchymal transition during cardiac development and oncogenic transformation. Genes Dev 2004, 18:99-115

48. Ma A, Boulton M, Zhao B, Connon C, Cai J, Albon J: A role for notch signaling in human corneal epithelial cell differentiation and proliferation. Invest Ophthalmol Vis Sci 2007, 48:3576-3585 